\title{
IRRITABLE BOWEL SYNDROME
}

\author{
Dr. Naeem Karnalkar
}

Homeopathic Consultant \& Medical Director, Star Poly Clinic, 160, old bazar, Khadki, Pune 411003

\begin{abstract}
The digestive tract starts from the mouth and ends up in the rectum and anal canal. Digestive signs can range from simple temporary conditions to chronic, enervating diseases. Persistent digestive complaints or severe symptoms warrant medical attention. Homeopathy has a very important role to play in the treatment of Gastrointestinal (GI) conditions. Anorexia, Nausea, vomiting, biliousness, hiccough, gastritis, heartburn, indigestion, constipation, Piles, irritable bowel syndrome (IBS), nervous disorders and many more conditions are treated successfully with homeopathy.

Modern and deskbound lifestyle, tensions, nervousness, stress and strain, irregular sleep pattern, eating habits, smoking and alcohol, and so many other factors are responsible to exacerbate the GI condition. (a) A brilliant student preparing for exams turns up to the doctor with anxiety and diarrhea. b) Held up in a traffic jam trying to reach in time, but couldn't, ends up his day with a bad stomach and abdominal distress. There are so many individual instances in the day to day affairs and the good news is that homeopathy has the cure. It goes deep into the system and treats the cause. It cures by raising the body's immunity, so it can resume its natural functions. Every case of GI condition calls for study of the patients individual symptoms, emotional and physical aspects. Ten patients suffering from IBS will receive ten different remedies.
\end{abstract}

\section{Irritable Bowel Syndrome (IBS)}

Irritable Bowel Syndrome also called colitis refers to a functional disorder of the colon or large intestine. It is an inflammatory disease, of unknown origin, characterised clinically by recurrent attacks of diarrhea with blood and mucus, left sided abdominal pain, heart burn, spasms and cramps and sense of abdominal distention, usually associated with constipation. It is more common in females between the ages $20-40$ years. Various factors like food intolerance, acute stress, anxiety may trigger the disease. Some people experience only mild symptoms of IBS. Others have intermittent episodes, where the symptoms come and go. Some of the patients may have severe and debilitating forms of the condition. Most of the symptoms of the IBS result from increased sensitivity of the intestines and movements of materials through the intestine either too quickly or too slowly. You may have one or more symptoms at any time. Possible aggravators of IBS may include stresses such as travel, social events, stressful events good or bad, certain foods and beverages including alcohol, carbonated drinks, high fat and greasy foods, change in work schedule, fructose(natural sugar in fruit), lactose(natural sugar in milk). The symptoms of IBS differ from person to person. Detail medical history is given by the patient which helps the doctor to analyse symptoms and decide treatment.

Fortunately long and protracted history of bowel symptoms without significant deterioration in general health is highly suggestive of IBS. Abdominal pain and diarrhea with mucus is one of the recognized manifestations of this disease. 
Case: A 45 years old man presented with a long history of IBS. For the last few months he had no control over his bowels, and would very often rush to the toilet and sometimes soil his clothes. He passed about 8 to 10 loose motions daily, sometimes with blood and mucus, and dyspepsia. Due to the fear of soiling his clothes and the urgent urge of going to the toilet he would be under great stress if he had to attend any social function or formal meetings at his office. Various homeopathic medications and different combinations were given to the patient and the first aim was to help him regain control over his bowel movements so that he gets confidence in his day to day activities. Within 6 weeks of initial treatment the frequency of bowels reduced to 4 to 5 per day. The patient was assured that this is a matter of time and in a few months he would slowly return to normal gastro intestinal functions. Soiling became rare after 12 weeks of treatment. After 6 months of treatment the frequency of stools reduced to 1 to 2 per day. Patient was advised to continue homeopathic treatment for at least 8 to 12 months. Some cases of IBS respond dramatically to the elimination of food intolerances. In this case the patient was advised to strictly stop drinking orange juice as he was drinking about 4 to 6 glasses of orange juice everyday.

Preventive aspects of IBS: Eat regularly scheduled meals and increase intake of fiber-rich foods, drink adequate fluids, avoid large meals, and avoid food items aggravating the condition, avoid stress, stress management exercises are very important, and meditation.

\section{Comments:}

It is important to see a doctor if a change in bowel habits lasts for more than 10 days. Before diagnosing IBS your physician will have considered and excluded other conditions particularly those affecting the colon by doing various tests like stool routine and culture, Barium enema, $\mathrm{X}$ rays and colonoscopy where ever necessary to rule out other digestive system disorders. Homeopathic medicines have been used extensively in the treatment of Gastro Intestinal Disorders and in particular IBS. Homeopathic treatment stresses on the factors that cause the disease and medicines are given to enhance digestion and reabsorption of the essential nutrients in the body.

Selection of the homeopathic remedy is a difficult task for the physician, because the remedy selected has to match closely the patient's symptoms. A constitutional remedy prescribed by an experienced professional is often the best approach to help the persons system regain its balance. Patients suffering from IBS have shown remarkable improvement with homeopathic medications and with the success rate of cure almost 90\%. Homeopathy has a good scope in IBS.

Dr. Naeem Karnalkar

Homeopathic Consultant \& Medical Director

Star Poly Clinic, 160, old bazar,

Khadki, Pune 411003, Tel: 9371357143

Web Site: www.drnaeem.110mb.com E-mail: drnaeemhk@yahoo.com

Hygeia.J.D.Med. February 2015

(c) All rights reserved.

Hygeia journal for drugs and medicines, 2229 3590, 09756221

DOI: 10.15254 / H.J.D.Med.7.2015.11 